\title{
C. BIDARD
}

\section{Le lemme de Sperner nu}

Revue française d'automatique, d'informatique et de recherche opérationnelle. Recherche opérationnelle, tome 32, n 2 (1998), p. 193-210.

<http://www.numdam.org/item?id=RO_1998_32_2_193_0>

(C) AFCET, 1998, tous droits réservés.

L'accès aux archives de la revue « Revue française d'automatique, d'informatique et de recherche opérationnelle. Recherche opérationnelle » implique l'accord avec les conditions générales d'utilisation (http://www.numdam.org/ legal.php). Toute utilisation commerciale ou impression systématique est constitutive d'une infraction pénale. Toute copie ou impression de ce fichier doit contenir la présente mention de copyright.

\section{Numdam}

Article numérisé dans le cadre du programme

Numérisation de documents anciens mathématiques

http://www.numdam.org/ 


\title{
LE LEMME DE SPERNER NU ( $\left.{ }^{\star}\right)$
}

\author{
par C. BIDARD $\left({ }^{1}\right)$
}

Communiqué par J.-Y. JAFFRAY

\begin{abstract}
Résumé. - Nous établissons plusieurs versions du lemme de Sperner. Elles sont dites nues en ce qu'elles valent pour des objets quelconques, non nécessairement géométriques, dès lors que les familles d'objets sont stables pour certaines opérations combinatoires. Les démonstrations d'existence sont constructives. Diverses applications sont examinées, notamment à la géométrie (hors du modèle simplicial d'origine) et à un problème de théorie économique. () Elsevier, Paris
\end{abstract}

Mots clés : Combinatoire, point fixe, Sperner.

Abstract. - Several versions of the Sperner lemma are established. They are called naked because they hold for any type of object, non necessarily of geometrical nature, provided that the families of objects are stable with regard to some combinatorial operations. The existence proofs are constructive. The results apply in particular to geometry (outside the initial simplicial model) and a problem of economic theory. (C) Elsevier, Paris

Keywords: Combinatorics, fixed point, Sperner.

\section{INTRODUCTION}

Nous $\left({ }^{2}\right)$ établissons plusieurs versions, dites nues, du lemme de Sperner. Ce qui distingue l'énoncé «nu » de la version géométrique est qu'un point y est un élément d'un ensemble abstrait, non nécessairement plongé dans $R^{n}$ : c'est une méthode de production dans certaines applications. De même les termes de sommet ou de face, s'ils témoignent de l'origine historique de ces lemmes, n'ont pas ici d'interprétation géométrique.

(*) Reçu en octobre 1995

( ${ }^{1}$ ) Université de Paris X-Nanterre, UFR de Sciences Économiques, 200, avenue de la République, 92001 Nanterre, France.

Adresse électronique : christian.bidard@u-paris10.fr

${ }^{2}$ Cet article est né d'une réflexion sur un travail commun avec Guido Erreygers. Je remercie également deux rapporteurs anonymes de la revue pour leurs commentaires et références.

Recherche opérationnelle/Operations Research, 0399-0559/98/02/

(C) Elsevier, Paris 
Dans sa version traditionnelle le lemme de Sperner géométrique (1928) est le moyen le plus direct pour établir l'important théorème de point fixe de Brouwer (1912). Étant donnée la décomposition d'un simplexe $\left[a_{1}, \ldots, a_{n}\right]$ de $R^{n}$, attribuons à chacun de ses sommets une marque, c'est-à-dire un chiffre de 1 à $n$ qui vérifie une condition de bord. Le lemme affirme l'existence d'au moins un sous-simplexe « bien marqué », dont les $n$ sommets portent toutes les marques de 1 à $n$. Il revient au même de dire que la famille $\mathcal{T}$ des ensembles de $n$ points bien marqués et la famille $\mathcal{C}$ des sous-simplexes ont une intersection non vide. C'est cette lecture qui est privilégiée, en constatant que les familles $\mathcal{T}$ et $\mathcal{C}$ sont stables pour certaines opérations, respectivement l'« insertion » et le « remplacement». L'idée fondamentale est que cette stabilité seule suffit à établir que l'intersection de deux familles abstraites quelconques est non vide, plus précisément contient un nombre impair d'éléments. Les démonstrations proposées sont constructives et s'inspirent de l'algorithme de Scarf (1967) qui fonde les méthodes modernes de calcul de point fixe.

La section 2 rappelle le principe de l'algorithme dans le cas géométrique. Bien que la connaissance de cette construction ne soit pas nécessaire pour s'assurer de la validité logique des raisonnements ultérieurs, elle est utile pour en suivre le fil conducteur. Puis le cadre géométrique est oublié et tous les théorèmes énoncés concernent, répétons-le, des points et éléments abstraits et non des objets géométriques. Ainsi pour le résultat de base (théorème 1) présenté en section 3. Appliqué au modèle simplicial, le théorème 1 présente deux faiblesses : il introduit une hypothèse spécifique sur la décomposition et ne fournit qu'une version non orientée du lemme. La section 4 énonce des conditions supplémentaires pour pallier ces défauts. Ces hypothèses étant vérifiées par le modèle simplicial, le lemme de Sperner géométrique apparaît au terme de cette construction comme l'application d'un énoncé abstrait à un support particulier. L'objet de la section 5 est de montrer que la version nue présente un intérêt propre en ce qu'elle sert à la résolution de problèmes pour lequel le lemme de Sperner géométrique n'est pas adapté. Un des exemples provient du problème de théorie économique qui est à l'origine de cette construction.

\section{LE MODÈLE SIMPLICIAL}

Considérons une décomposition simpliciale du simplexe unité $\left[a_{1}, \ldots, a_{n}\right]$ de $R^{n}$ et affectons à chaque sommet de la décomposition un chiffre de 1 à $n$ satisfaisant la condition suivante : tout point appartenant à un sous-simplexe 
$\left[a_{i_{1}}, \ldots, a_{i_{k}}\right]$ reçoit l'un des chiffres $i_{1}, \ldots$, ou $i_{k}$. En particulier la marque de $a_{i}$ est $i$. La figure 1 (avec $n=3$ ) obéit à l'hypothèse supplémentaire que la $(n-1)$-face $F_{0}=\left(a_{0}, \ldots, a_{n-1}\right)$ du simplexe initial n'est pas décomposée.

On définit un algorithme de recherche d'un simplexe bien marqué, c'està-dire qui porte tous les chiffres de 1 à $n$. La «porte » $F_{0}$ qui est marquée $1, \ldots, n-1$, ouvre dans la «salle » $C_{0}$. Sur la figure 1 celle-ci n'est pas bien marquée. Mais elle possède alors une seconde porte marquée $1, \ldots, n-1$ qui permet de pénétrer dans une nouvelle salle, etc. Puisque l'algorithme progresse tant qu'une salle bien marquée n'est pas atteinte, la clef de la démonstration de convergence est d'établir l'absence de cycle, ce qui est l'objet de l'argument de Lemke et Scarf (admettons provisoirement ce résultat). En conséquence il s'arrête dans une salle bien marquée, que nous appelons salon. Les propriétés suivantes, illustrées par la figure 1, ont une portée générale :

- l'algorithme converge, donc il existe au moins un salon. L'existence d'un nombre impair de salons est le lemme de Sperner dans la version correspondant au théorème 1 infra;

- plus précisément, le nombre de salons «blancs », c'est-à-dire dont l'orientation des sommets marqués $1,2, \ldots, n$ est la même que celle de $\left[a_{1}, \ldots, a_{n}\right]$, excède d'une unité celui des salons « noirs » d'orientation contraire (théorème 3 infra).

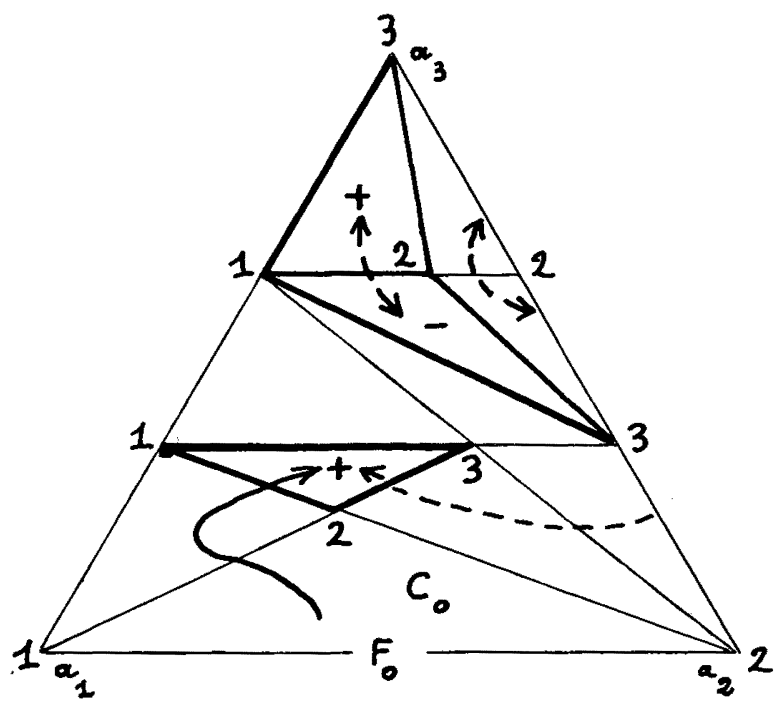

Figure 1. - L'algorithme géométrique. 
En quoi l'hypothèse de non-décomposition de la face $F_{0}=\left(a_{1}, \ldots, a_{n-1}\right)$ importe-t-elle? Comparons avec la face $\left(a_{2}, a_{3}\right)$ de la figure 1 , qui est décomposée. Un algorithme similaire est également défini en partant de toute « facette » incluse dans $\left(a_{2}, a_{3}\right)$ et marquée $(2,3)$. La difficulté illustrée par la figure 1 est qu'un tel algorithme risque finalement de " s'écraser » contre une autre facette de $\left(a_{2}, a_{3}\right)$. Ceci amène à distinguer deux objectifs :

- Si le but est d'établir le résultat d'imparité de Sperner, un argument par récurrence sur la dimension du simplexe suffit. En effet, la face $\left(a_{2}, a_{3}\right)$ est elle-même un simplexe de dimension inférieure et, par hypothèse de récurrence, admet un nombre impair de 2 -salons marqués $(2,3)$; pour certains d'entre eux l'algorithme peut certes s'écraser, mais ces 2-salons vont par paires (départ et arrivée sont permutables car l'algorithme fonctionne aussi en marche arrière!); reste un nombre impair de portes de départ, pour lesquelles l'algorithme s'achève dans autant de 3-salons distincts. A ceux-ci il faut ajouter les 3-salons du simplexe qui sont hors d'atteinte des portes de la face $\left(a_{2}, a_{3}\right)$, mais ceux-ci marchent aussi par paires (cf. fig. 1); au total l'imparité est établie pour la dimension supérieure et l'hypothèse de récurrence confirmée. Cet argument constitue l'essence de la preuve traditionnelle du lemme de Sperner (par exemple Berge, 1959). Ses éléments constitutifs se retrouveront dans les hypothèses du théorème 4 infra, qui énonce la version abstraite du lemme dépouillée du support géométrique et sans l'hypothèse de non-décomposition.

- S'il s'agit d'atteindre un salon, l'algorithme précédent ne suffit pas. En effet il ne garantit de trouver un $(k+1)$-salon que si tous les $k$-salons de la face $\left[a_{1}, \ldots, a_{k}\right]$ sont connus : rien n'assure qu'un $(k+2)$-salon sera localisé.

La démonstration constructive fait appel à une idée différente, due à Scarf (1967), et évite une récurrence sur la dimension. Elle consiste à plonger le simplexe $\left[a_{1}, \ldots, a_{n}\right]$ dans un simplexe plus grand. La partie raboutée est elle-même décomposée et marquée, mais de sorte qu'un bord du grand simplexe ne soit pas décomposé et que cette opération n'introduise aucun salon nouveau. Ceci ramène au cas examiné en premier lieu.

La version «nue » de ces résultats est fondée sur la remarque que l'algorithme repose fondamentalement sur deux propriétés :

- Étant donnés $n$ sommets marqués $1, \ldots, n$, tout autre point marqué $i$ peut être substitué à l'ancien sommet de même marque et un nouvel ensemble bien marqué est obtenu (famille $\mathcal{T}$ ). Dans le cadre «nu », la notion de marque est abandonnée au profit de la propriété plus générale d'insertion unique. 
- Sauf sur le bord, une face de dimension $n-1$ dans une décomposition simpliciale est commune à exactement deux simplexes de la décomposition $($ famille $\mathcal{C}$ ). En fait, il suffit que cette propriété vale pour les seules faces marquées $1, \ldots, n-1$.

\section{LA VERSION NUE DE BASE}

Nous abandonnons ici tout support géométrique et les points sont des objets mathématiques quelconques. Dans le cas du modèle simplicial le théorème 1 se réduit au lemme de Sperner avec non-décomposition d'une face $F_{0}$. Nous désignons par $k$-jeu un ensemble de $k$ points, par sommet un point considéré comme élément d'un $n$-jeu, enfin par face $n-1$ sommets d'un $n$-jeu. Pour faciliter la lecture la liste des $n$ sommets d'un $n$-jeu est notée entre crochets, celle des $n-1$ sommets d'un $(n-1)$-jeu entre parenthèses.

DÉFINITION 1 : Soient $N$ points $\left\{a_{1}, \ldots, a_{n} ; a_{n+1}, \ldots, a_{N}\right\}$. Un hôtel est défini par deux familles de $n$-jeux vérifiant les propriétés suivantes. Pour la famille $\mathcal{T}$ (les techniques) :

(i) $E_{0}=\left[a_{1}, \ldots, a_{n}\right] \in \mathcal{T}$.

(ii) Axiome d'insertion. Étant donnés une technique $T \in \mathcal{T}$ et un point, il existe une et une seule façon de substituer ce point à l'un des sommets de $T$ de façon à obtenir une nouvelle technique.

Pour la famille $\mathcal{C}$ (les candidats) :

(iii) Il existe exactement un candidat $C_{0}$ ayant pour face $F_{0}=$ $\left(a_{1}, \ldots, a_{n-1}\right)$.

(iv) Axiome de remplacement. Si un $(n-1)$-jeu, autre que $F_{0}$, est face d'un candidat et devient une technique après complétion par le point $a_{n}$, il est alors face d'exactement un autre candidat.

Un élément de $\mathcal{T} \cap \mathcal{C}$ est appelé salon.

THÉORÈME 1: Un hôtel admet un nombre impair de salons. En particulier $\mathcal{T} \cap \mathcal{C} \neq \varnothing$.

Démonstration: Nous appelons salle un candidat qui devient une technique après que $a_{n}$ a été substitué à l'un de ses sommets; une porte est une face d'une salle qui devient une technique après complétion par $a_{n}$. Notons que $C_{0}$ est une salle et $F_{0}$ une porte.

a) D'une salle à la suivante.

Soit une salle $S=\left[x_{1}, \ldots, x_{n}\right]$ et l'une de ses portes $\left(x_{1}, \ldots, x_{n-1}\right)$ c'està-dire que $\left[x_{1}, \ldots, x_{n-1}, a_{n}\right]$ est une technique. Si $\left(x_{1}, \ldots, x_{n-1}\right) \neq F_{0}$, 
l'axiome de remplacement implique l'existence d'un point unique $x_{n+1} \neq x_{n}$ tel que $S^{+}=\left[x_{1}, \ldots, x_{n-1}, x_{n+1}\right]$ soit un candidat. D'après (ii), le point $x_{n+1}$ peut être inséré d'une façon unique dans la technique $\left[x_{1}, \ldots, x_{n-1}, a_{n}\right]$, et une nouvelle technique est obtenue. S'il prend la place de $a_{n}, S^{+}$est une technique et, de plus, une salle. Si $x_{n+1}$ se substitue à, disons, $x_{n-1}$, alors $\left[x_{1}, \ldots, x_{n-2}, x_{n+1}, a_{n}\right] \in \mathcal{T}$. Donc $S^{+}$est un candidat qui devient une technique après que $a_{n}$ a pris la place de $x_{n-1}$, autrement dit c'est une salle. Dans les deux cas, étant données une salle $S$ et l'une de ses portes, une nouvelle salle $S^{+}$a été construite qui admet la même porte, et $S^{+}$est définie de façon unique (la seule exception concerne le couple $\left(C_{0}, F_{0}\right)$ : la condition (iii) énonce qu'aucune autre salle ne contient $\left.F_{0}\right)$.

b) Nombre de portes dans une salle.

- Deux portes au plus : Soit une salle $\left[x_{1}, \ldots, x_{n-1}, x_{n}\right]$ et l'une de ses portes $\left(x_{1}, \ldots, x_{n-1}\right)$. Considérons deux autres portes $\left(x_{1}, \ldots, x_{n-2}, x_{n}\right)$ et $\left(x_{1}, \ldots, x_{n-3}, x_{n-1}, x_{n}\right)$. Il en résulte que $T_{n}=\left[x_{1}, \ldots, x_{n-2}, x_{n-1}, a_{n}\right]$, $T_{n-1}=\left[x_{1}, \ldots, x_{n-2}, x_{n}, a_{n}\right]$ et $T_{n-2}=\left[x_{1}, \ldots, x_{n-3}, x_{n-1}, x_{n}, a_{n}\right]$ sont trois techniques. Dans $T_{n}, x_{n}$ peut donc être substitué soit à $x_{n-1}$ soit à $x_{n-2}$, ce qui contredit (ii). Une salle a donc deux portes au plus.

- Une porte dans un salon : soit $\left[x_{1}, \ldots, x_{n-1}, x_{n}\right]$ une technique et un candidat. $\mathrm{Si}\left(x_{1}, \ldots, x_{n-1}\right)$ et $\left(x_{1}, \ldots, x_{n-2}, x_{n}\right)$ étaient deux portes, alors $\left[x_{1}, \ldots, x_{n-1}, a_{n}\right]$ et $\left[x_{1}, \ldots, x_{n-2}, x_{n}, a_{n}\right]$ seraient toutes deux des techniques, et $a_{n}$ pourrait être inséré de deux façons dans la technique initiale, en contradiction avec (ii).

- Deux portes dans une salle qui n'est pas un salon: Soit $S=$ $\left[x_{1}, \ldots, x_{n-1}, x_{n}\right]$ une salle qui n'est pas une technique, $\left(x_{1}, \ldots, x_{n-1}\right)$ une porte, c'est-à-dire que $T=\left[x_{1}, \ldots, x_{n-1}, a_{n}\right]$ est une technique. Le point $x_{n}$ peut être inséré de façon unique dans $T$ et ne prend pas la place de $a_{n}$, sinon $S$ serait une technique. S'il prend la place de, disons, $x_{n-1}$, alors $\left[x_{1}, \ldots, x_{n-2}, x_{n}, a_{n}\right]$ est une technique. Donc $\left(x_{1}, \ldots, x_{n-2}, x_{n}\right)$ est une autre porte de $S$.

c) L'argument de Lemke et Scarf.

L'argument de Lemke (1965) et Scarf (1967) s'applique à l'hôtel. $F_{0}$ est la porte d'entrée d'un candidat $C_{0}$, qui est une salle. Si $C_{0}$ n'est pas une technique il admet une unique autre porte $F_{1}$ qui communique avec une nouvelle salle $C_{0}^{+}=C_{1}$. Tant qu'une technique n'est pas atteinte, l'argument est répété, la marche d'une salle à la suivante étant définie de façon unique. S'il existait un cycle, considérons la première salle $S$ dans lequel on revient. Puisque les deux salles adjacentes ont déjà été explorées à l'occasion de la 
première visite, et qu'il s'agit des seules entrées de $S$, une telle première salle n'existe pas. En l'absence de cycle, la marche s'arrête donc dans une dernière salle qui, n'ayant qu'une porte, est un salon $S_{0}$. Donc $\mathcal{T} \cap \mathcal{C} \neq \varnothing$.

Soit $S_{1} \neq S_{0}$ un autre élément de $\mathcal{T} \cap \mathcal{C}$, s'il en existe. Choisissons $S_{1}$ comme point de départ d'une autre marche. $S_{1}$ a une seule porte. Le nouveau chemin ne croise pas l'ancien, car deux chemins ayant une salle commune coïncident puisque chaque salle a deux portes au plus. Ce chemin s'achève dans un autre salon $S_{2}$. Un nouveau couple a ainsi été défini, où $S_{1}$ et $S_{2}$ sont les salons initial et final d'un autre chemin. Puisque les salons autres que $S_{0}$ viennent par paires, $\mathcal{T} \cap \mathcal{C}$ a un nombre impair de salons.

Comme signalé par un rapporteur, le théorème 1 repose sur des hypothèses analogues à celles de Tuy (1979) (avec énoncé d'existence, sans référence à l'imparité), qui renvoie lui-même aux résultats établis indépendamment par Todd (1974) et Gould et Tolle (1974). Tous ces auteurs s'attachent à l'analyse numérique et visent à intégrer dans un cadre conceptuel unique un ensemble d'algorithmes qui font appel à une méthode du pivot et sont utilisés dans divers problèmes de complémentarité, d'optimisation ou de point fixe. La variante suivante du théorème 1 est d'énoncé plus simple et de démonstration identique (celle-ci est laissée au lecteur), sans constituer un résultat d'existence :

THÉORÈME 2 : Soient $N$ points $\left\{a_{1}, \ldots, a_{N}\right\}$ et deux familles $\mathcal{T}^{\prime}$ et $\mathcal{C}^{\prime}$ de n-jeux. Supposons que:

- Étant donnés $T \in \mathcal{T}^{\prime}$ et un point, il existe une et une seule façon de substituer ce point à un sommet de $T$ de façon à obtenir un nouveau membre de $\mathcal{T}^{\prime}$.

- Si un $(n-1)$-jeu est face d'un membre de $\mathcal{C}^{\prime}$ et appartient à $\mathcal{T}^{\prime}$ après complétion par le point $a_{n}$ il est aussi face d'exactement un autre membre de $\mathcal{C}^{\prime}$.

Alors $\mathcal{T}^{\prime} \cap \mathcal{C}^{\prime}$ admet un nombre pair d'éléments.

\section{EXTENSIONS DU LEMME NU}

Le modèle simplicial constitue à la fois le support d'origine et l'application la plus directe du théorème 1 . Lorsque les points $a_{i}$ de la définition 1 sont des points de $R^{n},\left[a_{1}, \ldots, a_{n}\right]$ le simplexe unité, $\mathcal{C}$ une décomposition simpliciale de sommets $\left\{a_{1}, \ldots, a_{n} ; a_{n+1}, \ldots, a_{N}\right\}$ et enfin qu'une marque est attachée à chaque point, le théorème 1 affirme l'existence d'un nombre impair de simplexes bien marqués si l'axiome (iii) est vérifié, c'est-à-dire si 
l'un des simplexes de la décomposition a pour face $F_{0}=\left(a_{1}, \ldots, a_{n-1}\right)$. L'énoncé du lemme de Sperner traditionnel se dispense de cette dernière restriction. Par ailleurs ce lemme admet une version orientée plus élaborée.

Nous nous proposons d'intégrer ces deux résultats à la version nue. L'opération fait appel à des hypothèses supplémentaires qui sont effectivement vérifiées par le modèle simplicial. Ces deux extensions sont indépendantes. Nous commençons par la question de l'orientation, en conservant la restriction mentionnée sur la décomposition. Les démonstrations sont constructives en ce qu'elles désignent un chemin pour atteindre un sous-simplexe bien marqué.

\subsection{Le lemme orienté}

Nous portons désormais attention à l'ordre dans lequel les sommets d'un $n$-jeu sont considérés : lorsqu'un point est inséré dans une technique ou un candidat, il prend la place du sommet auquel il se substitue. Toutefois, cet ordre n'est défini qu'à une substitution paire près sur les sommets. Un $n$-jeu ordonné est désigné par une flèche. Des techniques ou des candidats voisins ne diffèrent que par un sommet.

DÉfINITION 2: Une famille $\mathcal{T}_{\text {or }}$ de techniques ordonnées est orientable si, lorsque l'ensemble non ordonné des sommets d'une technique est globalement préservé après une suite d'insertions, la technique ordonnée finale diffère de l'initiale par une substitution paire sur les sommets. Alors la famille $\mathcal{T}_{\text {or }}$ est orientée, c'est-à-dire qu'il existe une fonction sgt : $\mathcal{T}_{\text {or }} \rightarrow\{-1,+1\}$ telle que :

. La technique $\vec{E}_{0}=\left[a_{1}, \ldots, a_{n}\right]$ reçoit la valeur $\operatorname{sgt}\left(\vec{E}_{0}\right)=+1$.

. Pour toute substitution $\tau$ sur les sommets d'une technique $\vec{T}$

$$
\operatorname{sgt}(\tau(\vec{T}))=\operatorname{sign}(\tau) \operatorname{sgt}(\vec{T})
$$

avec $\operatorname{sign}(\tau)=+1$ ou -1 selon la parité de la substitution $\tau$.

. La fonction sgt assigne la même valeur à deux techniques voisines.

Définitron 3 : Une famille $\mathcal{C}_{\text {or }}$ de candidats ordonnés est orientable si, lorsque l'ensemble des sommets d'un candidat est globalement préservé après une suite de remplacements, la parité de la substitution finale sur les sommets est celle du nombre de remplacements. Alors la famille $\mathcal{C}_{\text {or }}$ est orientée, c'est-à-dire qu'il existe une fonction sgc : $\mathcal{C}_{\text {or }} \rightarrow\{-1,+1\}$ telle que :

. Le candidat $\vec{C}_{0}=\left[a_{1}, \ldots, a_{n-1}, a_{n^{*}}\right]$ reçoit la valeur $\operatorname{sgc}\left(\vec{C}_{0}\right)=+1$. 
. Pour toute substitution $\sigma$ sur les sommets d'un candidat $\vec{C}$

$$
\operatorname{sgc}(\sigma(\vec{C}))=\operatorname{sign}(\sigma) \operatorname{sgc}(\vec{C})
$$

avec $\operatorname{sign}(\sigma)=+1$ ou -1 selon la parité de la substitution $\sigma$.

. La fonction sgc assigne des valeurs opposées à deux candidats voisins.

DÉFINITION 4 : Un hôtel orienté est défini par une famille orientée $\mathcal{T}_{\text {or }}$ de techniques et une famille orientée $\mathcal{C}_{\text {or }}$ de candidats qui satisfont les axiomes posés dans la définition 1. Pour un salon la valeur $\operatorname{col}=\operatorname{sgt}(\vec{T}) \operatorname{sgc}(\vec{C})$ est indépendante de l'ordre sur ses sommets. Le salon est blanc si col $=+1$ et noir si $\mathrm{col}=-1$.

THÉORÈme 3 : Dans un hôtel orienté il y a un salon blanc de plus que de salons noirs.

Démonstration: Reprenons la démonstration de la version non orientée. Le point de départ est constitué de la technique $\vec{E}_{0}=\left[a_{1}, \ldots, a_{n}\right]$ et du candidat $\vec{C}_{0}=\left[a_{1}, \ldots, a_{n-1}, a_{n^{*}}\right]$ avec $\operatorname{sgt}\left(\vec{E}_{0}\right)=\operatorname{sgc}\left(\vec{C}_{0}\right)=+1$. Après un certain nombre d'étapes, la situation est décrite par un couple $(\vec{T}, \vec{C})$ possédant les propriétés $(\mathrm{P}): \vec{T}=\left[x_{1}, \ldots, x_{i-1}, a_{n}, x_{i+1}, \ldots, x_{n}\right]$ est une technique, $\vec{C}=\left[x_{1}, \ldots, x_{i}, \ldots, x_{n}\right]$ un candidat, la porte d'entrée pour $\vec{C}$ était $\left(x_{1}, \ldots, x_{i-1}, x_{i+1}, \ldots, x_{n}\right)$ et $\operatorname{sgt}(\vec{T})=\operatorname{sgc}(\vec{C})$. Si $C$ est une technique, il s'agit d'un salon blanc. Sinon ce candidat admet une porte de sortie $\left(x_{1}, \ldots, x_{j-1}, x_{j+1}, \ldots, x_{n}\right)$ avec $j \neq i$ et un nouveau candidat s'écrit $\vec{C}^{+}=\left[x_{1}, \ldots, x_{j-1}, x_{j^{*}}, x_{j+1}, \ldots, x_{n}\right]$ avec $\operatorname{sgc}\left(\vec{C}^{+}\right)=-\operatorname{sgc}(\vec{C})$. Insérons $x_{j^{*}}$ dans $\vec{T}$ :

- Si $x_{j^{*}}$ prend la place de $a_{n}$, qui est en $i$-ième position, la nouvelle technique $\vec{T}^{*}$ coïncide avec $\vec{C}^{+}$à permutation près de $x_{j^{*}}$ et $x_{i}$. Donc $C^{+}$ est une technique, avec

$$
\operatorname{sgt}\left(\vec{C}^{+}\right)=-\operatorname{sgt}\left(\vec{T}^{*}\right)=-\operatorname{sgt}(\vec{T})=-\operatorname{sgc}(\vec{C})=\operatorname{sgc}\left(\vec{C}^{+}\right),
$$

c'est-à-dire que $C^{+}$est un salon blanc.

- Si $x_{j^{*}}$ prend la place de $x_{k}$, alors

$$
\vec{T}^{*}=\left[x_{1}, \ldots, x_{i-1}, a_{n}, x_{i+1}, \ldots, x_{k-1}, x_{j^{*}}, x_{k+1}, \ldots, x_{n}\right]
$$

est une technique. Après permutation de $a_{n}$ et $x_{j^{*}}$ dans $\vec{T}^{*}$, une nouvelle technique ordonnée $\vec{T}^{+}$est obtenu, avec

$$
\operatorname{sgt}\left(\vec{T}^{+}\right)=-\operatorname{sgt}\left(\vec{T}^{*}\right)=-\operatorname{sgt}(\vec{T})=-\operatorname{sgc}(\vec{C})=\operatorname{sgc}\left(\vec{C}^{+}\right) .
$$


Le nouveau couple $\left(\vec{T}^{+}, \vec{C}^{+}\right)$a les mêmes propriétés (P) que le précédent. Donc, lorsque la marche partie de $\left(\vec{E}_{0}, \vec{C}_{0}\right)$ arrive à son terme, le salon final qui est atteint est blanc.

Si l'algorithme part d'un salon noir le couple de départ $(\vec{T}, \vec{C})$ qui s'adapte au raisonnement précédent est formé de cette technique orientée $\vec{T}$ et de l'unique candidat $\vec{C}$ avec laquelle elle a une porte commune. Ce couple est apparié, c'est-à-dire que $\operatorname{sgt}(\vec{T})=\operatorname{sgc}(\vec{C})$, et le salon final sera blanc. Si le départ est un salon blanc, la marche s'achève dans un salon noir. D'où le résultat.

\subsection{Le lemme de la tour}

Il s'agit de se débarrasser ici de l'hypothèse (iii) du théorème 1. Cette hypothèse est toutefois vérifiée pour $n=1$. Par ailleurs, pour le modèle simplicial, une face d'un simplexe est elle-même un simplexe de dimension inférieure, d'où l'idée d'accéder par récurrence à la dimension $n$. Les axiomes ci-dessous sont effectivement satisfaits par le modèle simplicial lorsque $P_{i}$ désigne l'ensemble des sommets de la décomposition situés sur la face $\left[a_{1}, \ldots, a_{i}\right]$ du simplexe.

Définition 5 : Soient $N$ points $P=\left\{a_{1}, \ldots, a_{n}, a_{n+1}, \ldots, a_{N}\right\}$ et $n$ sous-ensembles $P_{1}=\left\{a_{1}\right\} \subset P_{2} \subset \ldots \subset P_{n}=P$ avec $a_{i} \in P_{i} \backslash P_{i-1}$. Une tour est donnée par deux familles $\mathcal{T}$ et $\mathcal{C}$ de $n$-jeux tels que :

- Pour la famille $\mathcal{T}$ des techniques :

$\left(\mathrm{G}_{1}\right) \quad\left[a_{1}, \ldots, a_{n}\right] \in \mathcal{T}$.

$\left(\mathrm{G}_{2}\right) \quad \forall i=1, \ldots, n$ une technique a au plus $i$ sommets dans $P_{i}$.

$\left(\mathrm{G}_{3}\right)$ L'axiome d'insertion est vérifié.

- Pour la famille $\mathcal{C}$ des candidats :

$\left(\mathrm{H}_{1}\right) \quad$ Il existe au moins un candidat de sommet $a_{1}$.

$\left(\mathrm{H}_{2}\right) \quad \forall i=1, \ldots, n$ un candidat a au plus $i$ sommets dans $P_{i}$.

$\left(\mathrm{H}_{3}\right)$ Soient $i$ points $\left(x_{1}, \ldots, x_{i}\right), 1 \leq i \leq n-1$, qui appartiennent à un candidat $C \in \mathcal{C}$ et tels que $\left[x_{1}, \ldots, x_{i}, a_{i+1}, \ldots, a_{n}\right] \in \mathcal{T}$ :

a) Si $\left(x_{1}, \ldots, x_{i}\right) \subset P_{i}$, il existe un unique $x_{i+1}$ dans $P_{i+1}$ tel que $\left(x_{1}, \ldots, x_{i}, x_{i+1}\right)$ est sous-ensemble d'un candidat $C \in \mathcal{C}$.

b) $\mathrm{Si}\left(x_{1}, \ldots, x_{i}\right) \not \subset P_{i}$ et qu'il existe $x_{i+1}$ tel que $\left(x_{1}, \ldots, x_{i}, x_{i+1}\right) \subset$ $P_{i+1}$ et est sous-ensemble d'un certain candidat $C \in \mathcal{C}$, il existe exactement un autre point $x_{i+1}^{\prime}$ de $P_{i+1}$ qui possède les mêmes propriétés. 
THÉORÈME 4 : Une tour admet un nombre impair de salons.

Le théorème 4 peut s'établir par récurrence sur la dimension, conformément à l'idée qui a présidé au choix des axiomes. Nous avons mentionné en section 2 les limites de cette approche. La démonstration ci-dessous s'inspire de la méthode constructive de Scarf à laquelle la figure 2 apporte un support visuel dans le cadre du modèle simplicial : l'adjonction d'une « jupe » permet de se ramener aux conditions d'application du théorème 1. L'idée s'avère généralisable au cadre abstrait.

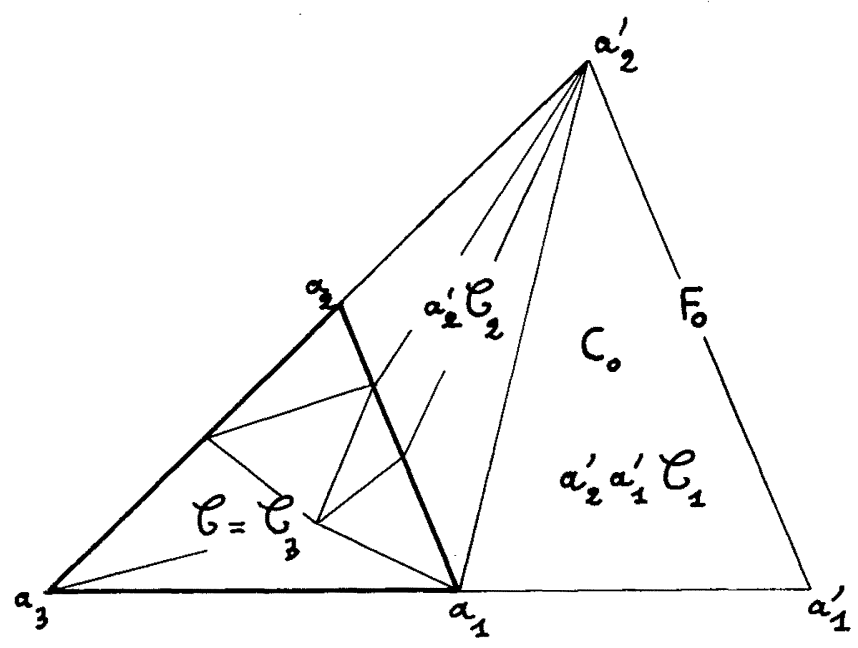

Figure 2. - Simplexe avec jupe.

Démonstration (indications) : Introduisons $n-1$ points supplémentaires $a_{1}^{\prime}, \ldots, a_{n-1}^{\prime}$ et définissons $P^{\prime}=P \cup\left\{a_{1}^{\prime}, \ldots, a_{n-1}^{\prime}\right\}$. Soit $\mathcal{T}^{\prime}$ la collection des $n$-jeux sur $P^{\prime}$ qui appartiennent à $\mathcal{T}$ lorsque chaque sommet $a_{i}^{\prime}$ est remplacé par $a_{i}$. Désignons par $\mathcal{C}_{n-j}$ les $(n-j)$-uples $\left(x_{1}, \ldots, x_{n-j}\right)$ traces sur $P_{n-j}$ des $n$-uples de $\mathcal{C}$, et par $\left\{a_{n-1}^{\prime} \ldots a_{n-j}^{\prime} \mathcal{C}_{n-j}\right\}$ les $n$-uples obtenus en les complétant par les points $a_{n-j}^{\prime}$ à $a_{n-1}^{\prime}$. Soit enfin $\mathcal{C}^{\prime}$ la famille $\mathcal{C}^{\prime}=\mathcal{C}_{n} \cup\left\{a_{n-1}^{\prime} \mathcal{C}_{n-1}\right\} \cup \ldots \cup\left\{a_{n-1}^{\prime} \ldots a_{n-j}^{\prime} \mathcal{C}_{n-j}\right\} \cup \ldots \cup\left\{a_{n-1}^{\prime} \ldots a_{1}^{\prime} a_{1}\right\}$ (fig. 2 pour $n=3)$. En posant $F_{0}=\left(a_{n-1}^{\prime}, \ldots, a_{1}^{\prime}\right)$ et $C_{0}=$ $\left[a_{n-1}^{\prime}, \ldots, a_{1}^{\prime}, a_{1}\right]$ un $\mathcal{T}^{\prime} \mathcal{C}^{\prime}$-hôtel est construit, auquel le théorème 1 s'applique. Après transformation de $a_{i}^{\prime}$ en $a_{i}$ les sommets d'un $n$-jeu de $\mathcal{C}^{\prime} \backslash \mathcal{C}_{n}$ deviennent $n$ points de $P_{n-1}$, donc un tel $n$-jeu n'appartient pas à $\mathcal{T}^{\prime}$ d'après $\left(\mathrm{G}_{2}\right)$. Ainsi il existe un nombre impair d'éléments dans $\mathcal{T}^{\prime} \cap \mathcal{C}^{\prime}=\mathcal{T}^{\prime} \cap \mathcal{C}_{n}=\mathcal{T}^{\prime} \cap \mathcal{C}=\mathcal{T} \cap \mathcal{C}$. 


\section{APPLICATIONS}

\subsection{Le lemme de Sperner géométrique}

Le lemme de Sperner géométrique et la preuve algorithmique de Scarf ont guidé la recherche précédente. Par rapport au théorème 1 , le théorème 3 introduit une orientation des simplexes bien marqués et le théorème 4 se dispense de l'hypothèse restrictive selon laquelle une face du simplexe n'est pas décomposée. Stricto sensu, l'énoncé général du lemme de Sperner (existence de $k+1$ simplexes bien marqués de sens positif et de $k$ de sens négatif) ne résulte pas des théorèmes 3 et $4:$ il faudrait encore combiner les deux arguments en reprenant la démonstration du théorème 4 et en montrant que les orientations de $\mathcal{T}$ et de $\mathcal{C}$ s'étendent à des orientations de $\mathcal{T}^{\prime}$ et $\mathcal{C}^{\prime}$. Cet exercice est laissé au lecteur.

Le lemme de Sperner géométrique est un résultat profond qui implique le théorème de point fixe de Brouwer, le lemme KKM (Knaster-KuratowskiMazurkiewicz, 1929) et est lié au concept de degré topologique : tous ces résultats sont en fait équivalents (Yoseloff, 1974; Lasry et Robert, 1975; voir Border, 1985, pour un exposé détaillé). La version nue met à jour les éléments fondamentaux de cette structure. Ainsi, parce qu'elle ne distingue pas entre l'existence et l'imparité, elle suggère qu'en ce domaine tout résultat s'étend en un résultat d'imparité : c'est précisément l'objet spécifique du travail de Le Van (1982) sur le degré topologique.

\subsection{Orientation}

En revanche la séparation des solutions selon leur couleur fait appel à une hypothèse supplémentaire relative à la possibilité d'orientation. Celleci est satisfaite par le modèle simplicial : la fonction sgt associe à toute technique ordonnée le nombre +1 ou -1 selon la parité de la substitution entre les marques de ses sommets ordonnés et l'ordre naturel $1, \ldots, n$; la fonction sgc associe à tout candidat ordonné le signe du déterminant des $n$ vecteurs de $R^{n}$ dont les extrémités sont les sommets du candidat. Dans le cadre général, abstrait et non géométrique, deux types de difficultés sont susceptibles d'apparaître dans l'application du théorème 3. Parfois une orientation existe mais la famille $\mathcal{T}$ est non connexe : l'orientation n'est définie de façon unique que sur la composante connexe de $\vec{E}_{0}$. Un exemple de famille non connexe avec $n=4$ et $N=7$ points (nous écrivons le chiffre $i$ à la place de $a_{i}$ ) est

$$
\mathcal{T}_{1}=\{1234,1235,1236,1237,1567,2567,3567,4567\} .
$$


Le lecteur vérifiera que cette famille vérifie bien les axiomes (i) et (ii) (chaque « chiffre » peut être inséré de façon unique dans tout "nombre » de façon à obtenir un autre nombre de la liste). Comme les quatre premières techniques ont les trois sommets 1, 2, 3 en commun mais que les quatre dernières n'en ont qu'un, il est impossible de passer par insertion d'une sousfamille à l'autre et $\mathcal{T}_{1}$ a donc deux composantes connexes. Le théorème 3 reste toutefois valide quelle que soit l'orientation choisie sur les composantes autres que celle de $\vec{E}_{0}$. La difficulté est plus sérieuse quand une orientation n'existe pas : ainsi pour $n=4$ et $N=7$ et la famille de techniques

$$
\begin{aligned}
\mathcal{T}_{2}= & \{1234,7234,7534,7564,2564,2164, \\
& 7164,7163,7263,5263,5163,5143\} .
\end{aligned}
$$

En respectant l'ordre dans l'insertion, les six premiers nombres montrent comment 1234 est transformé en 2164. Après insertion du chiffre 3, 2164 devient 2134 qui diffère de 1234 par une permutation. Donc cette famille n'est pas orientable. Incidemment, l'existence même de familles non orientables établit que le lemme nu n'est pas réductible à un modèle géométrique.

\subsection{La notion d'homotopie formelle}

Les propriétés d'une famille de candidats peuvent être étudiées en ellesmêmes. Considérons ainsi la famille $\mathcal{C}=\{124,234,314,125,235,315\}$. Appliquons les règles suivantes "d'homotopie formelle » à tout lacet $\{1 a b \ldots y z 1\}$ qui suit les arêtes de $\mathcal{C}: k l m=k m$ si et seulement si $k l m$ appartient à $\mathcal{C}$, et $k l k=k k=k$. Alors tout lacet est réductible à un seul point, donc le « groupe d'homotopie formelle » de $\mathcal{C}$ est trivial. C'est ici le cas parce que la famille $\mathcal{C}$ décrit la décomposition simpliciale d'une sphère représentée par un équateur de sommets $1,2,3$ et deux pôles 4 et 5 . En revanche une famille $\mathcal{C}^{\prime}$ représentant la décomposition d'un tore aurait un groupe non trivial. L'objet de l'homotopie formelle est d'étudier les familles de candidats indépendamment de tout support géométrique, avec l'idée de tirer l'homologie ou l'homotopie vers la combinatoire pure.

\subsection{Suites techniques}

Dans le cadre abstrait, la façon générale pour étudier la validité de l'axiome d'insertion (dans la version non orientée de la définition 1) consiste à repérer chaque point par un «chiffre » de 1 à $N$ (ne pas confondre avec la marque du modèle simplicial qui a ses valeurs dans $1, \ldots, n$ ) et chaque technique par le «nombre » formé de la liste non ordonnée des $n$ chiffres de ses 
sommets : ainsi pour $\mathcal{T}_{1}$ et $\mathcal{T}_{2}$ de la section 5.2. L'axiome d'insertion est vérifié si et seulement si la collection de nombres est une « suite technique » :

DÉFInItion 6 : Une suite technique $S$ est une collection de nombres telle que tout chiffre peut être inséré de façon unique dans un nombre de $S$ de façon à former un nombre de $S$.

Les suites techniques peuvent être examinées pour elles-mêmes : par exemple, pour $n=3$ et $N=6$, leur liste exhaustive (à permutation près sur les chiffres) est

$$
\begin{aligned}
& S_{1}=\{123,124,125,126\} \\
& S_{2}=\{123,124,126,153,154,156\} \\
& S_{3}=\{135,136,145,146,235,236,245,246\} \\
& S_{4}=\{123,234,345,451,512,623,456,562\} \\
& S_{5}=\{123,234,345,451,512,613,635,652,624,641\}
\end{aligned}
$$

Les suites $S_{1}, S_{2}$ et $S_{3}$ sont factorisables, au sens où $S_{3}=\{i j k ; i=1$ ou $2, j=3$ ou $4, k=5$ ou 6$\}$. Dans le lemme de Sperner géométrique tous les sommets de même marque sont parfaitement substituables les uns aux autres dans la famille des $n$-jeux bien marqués et la suite technique est factorisable. Ce n'est pas nécessairement le cas pour le lemme nu qui en ce sens est plus général que le lemme habituel. C'est ce qu'illustrent les deux applications suivantes.

\subsection{Application géométrique}

Dans $R^{n}$ considérons $N$ vecteurs $u_{i}(i=1, \ldots, N, N \geq n)$ et, indépendamment, une direction $d$. Ces données sont en position générique. On dira que le $n$-jeu $\left[u_{i_{1}}, \ldots, u_{i_{n}}\right]$ est une technique si et seulement si $d$ est obtenu par combinaison positive des vecteurs $u_{i_{1}}, \ldots, u_{i_{n}}$. Le lecteur vérifiera que l'axiome d'insertion est satisfait (le résultat est géométriquement intuitif pour $n=2$ ou 3). En général la suite technique associée n'est pas factorisable. Cette famille $\mathcal{T}$ est orientée. Toujours dans le cadre géométrique, l'axiome de remplacement (oublions la restriction sur $F_{0}$ ) est également détachée du modèle simplicial dans la configuration suivante : étant donnés $N$ vecteurs $v_{i}$ en position générique dans $R^{n}$, considérons leur enveloppe convexe $K$; on dit que $\left[v_{i_{1}}, \ldots, v_{i_{n}}\right]$ est un candidat s'il constitue une $n$-face de $K$. Cette famille $\mathcal{C}$ est orientée. 
En combinant ces deux familles, il résulte du théorème 2 qu'il existe génériquement un nombre pair de $n$-uples $\left(i_{1}, \ldots, i_{n}\right)$ d'indices tels que les vecteurs $u_{i}$ correspondants appartiennent à $\mathcal{T}$ et les vecteurs $v_{i}$ à $\mathcal{C}$.

\subsection{Application économique}

La dernière application utilise ces deux mêmes familles (avec une face particulière $F_{0}$ ) et se fonde sur le lemme nu pour analyser les solutions d'un problème de théorie économique.

Le problème relève de la théorie des prix de production. Il y a $n$ biens, du travail homogène et $N$ méthodes de production. Pour $i=1, \ldots, n$, la $i$-ième méthode est l'élimination libre du bien $i$ : ce bien figure seul du côté des intrants et il n'y a aucun produit. Chacune des $N-n$ dernières méthodes de production est décrite par le vecteur-ligne $a_{i} \in R_{+}^{n}$ des biens qui entrent dans le processus conjointement à une unité de travail $\left(l_{i}=1\right)$ et par le vecteur-ligne $b_{i} \in R_{+}^{n}$ des biens produits. Les rendements sont constants (si l'on multiplie les intrants par de la $i$-ième méthode par le scalaire $y_{i}>0$, les produits le sont aussi). $(A, l, B)$ est la matrice $N \times(n+1+n)$ décrivant les méthodes disponibles, obtenue en empilant les vecteurs-lignes $\left(a_{i}, l_{i}, b_{i}\right)$. Du côté des quantités, l'exigence est que le produit net de l'économie soit un vecteur $d$ donné, représentatif de la demande de consommation des agents. Autrement dit le vecteur-ligne semi-positif $y=\left(y_{1}, \ldots, y_{N}\right)>0$ des niveaux d'activité doit être tel que

$$
y(B-A)=d .
$$

Par ailleurs les prix $p \in R_{+}^{n}$ sont tels que toutes les méthodes utilisées procurent le même taux de profit donné $r \geq 0$, tandis que les autres méthodes rapportent moins. En prenant le salaire pour unité de compte, l'exigence de profitabilité s'écrit sous forme d'inégalité vectorielle avec complémentarité

$$
B p \leq(1+r)(A p+l) \quad[y]
$$

puisque $y_{i}\left(a_{i} p+l_{i}\right)$ représente le coût de production et $y_{i} b_{i} p$ les recettes de la méthode $i$. La question est d'étudier les solutions du système (1)-(2) dont les inconnues sont les niveaux d'activité $y>0$ et les prix $p>0$.

THÉORÈme 5: Sous l'hypothèse

$$
\exists y>0, \quad y(B-(1+r) A) \gg 0
$$

le système (1)-(2) admet génériquement un nombre impair de solutions. Plus précisément chaque solution est génériquement constituée de $n$ méthodes 
actives $\left(a_{i}, l_{i}\right) \rightarrow b_{i}$ (c'est-à-dire avec un niveau d'activité $y_{i}$ positif) et le nombre de solutions pour lesquelles $\operatorname{det}\left(\ldots, b_{i}-(1+r) a_{i}, \ldots\right)$ et $\operatorname{det}\left(\ldots, b_{i}-a_{i}, \ldots\right)$ ont même signe excède d'une unité celui des solutions à signes contraires.

Démonstration (indications) : Les axes de l'argumentation sont :

- Remplaçons provisoirement la condition (1) par

$$
\exists \lambda, \quad \exists y>0, \quad y(B-A)=\lambda d
$$

sans imposer $\lambda>0$. Génériquement une solution de $\left(1^{\prime}\right)$-(2) est composée de $n$ méthodes actives : en effet la direction $d$ n'est pas (génériquement) obtenue par combinaison de moins de $n$ vecteurs $b_{i}-a_{i}$, et par ailleurs le vecteur-prix ne satisfait pas (génériquement) plus de $n$ égalités du type $(1+r)\left(a_{i} p+l_{i}\right)=b_{i} p$.

- Les combinaisons de $n$ méthodes qui satisfont la condition $\left(1^{\prime}\right)$ vérifient l'axiome d'insertion, comme relevé dans la sous-section 5.5. Cet ensemble n'est pas vide car la condition $\left(1^{\prime}\right)$ est satisfaite par la combinaison formée des $n$ méthodes d'élimination libre. Ainsi les $n$-jeux de méthodes compatibles avec $\left(1^{\prime}\right)$ forment une famille de techniques au sens de la définition 1.

- Passons à l'étude de la condition (2). Considérons la fermeture convexe $K^{\prime}$ de l'ensemble des vecteurs $v_{i}=b_{i}-(1+r) a_{i}$ pour $i=n+1, \ldots, N$ et de l'orthant négatif ( $K^{\prime}$ est l'ensemble des vecteurs du type $y(B-(1+r) A)$ pour $y>0$ et $y l=1$, c'est-à-dire avec une unité de travail dans l'économie; par rapport à la section précédente, l'intervention de l'orthant négatif tient à l'élimination libre). Un $n$-uple de méthodes est compatible avec la condition de profitabilité (2) si et seulement si ces méthodes correspondent aux sommets d'une $n$-face de $K^{\prime}$, le vecteur-prix $p$ associé étant orthogonal à la face (l'équation de cette face étant $x p=1$ ). Par ailleurs $K^{\prime}$ admet une face $C_{0}$ composée d'une méthode ordinaire et de l'élimination libre des biens 1 à $n-1$. On vérifie que les $n$-jeux de méthodes compatibles avec la condition (2) forment une famille de candidats au sens du théorème 1.

- Le théorème 1 établit l'existence d'un nombre impair de solutions du système $\left(1^{\prime}\right)-(2)$. La condition $(\mathrm{H})$ est alors utilisée pour montrer que, pour toute solution, $\lambda$ est positif; une homothétie sur les niveaux d'activité ramène à $\lambda=1$, donc aux solutions du système (1)-(2). Et comme les familles de techniques et de candidats considérées dans cette démonstration sont orientées, les fonctions sgt et sgc étant définies par les signes de déterminants, le théorème 3 permet de conclure comme indiqué (voir Bidard, 1995, pour les détails techniques). 
Le modèle économique lui-même est dû à Lippi (1979) qui a établi l'existence d'une solution. L'imparité a d'abord été obtenue par des moyens spécifiques par Bidard et Erreygers (1995). La référence au théorème 1 montre que l'hypothèse de demande fixe ne joue ici que par l'axiome d'insertion et pourrait être remplacée par toute autre pour laquelle la propriété serait maintenue. Dans le cadre de la théorie de l'équilibre général, des résultats similaires d'imparité ont été obtenus sous l'hypothèse de différentiabilité (Dierker, 1972).

\section{CONCLUSION}

Nous avons établi diverses versions du lemme de Sperner, toutes constructives, qui sont dites nues en ce qu'elles valent pour des familles d'objets quelconques vérifiant des propriétés de stabilité à l'égard de certaines opérations. Ceci permet d'appliquer le lemme hors du cadre où il est habituellement énoncé ou, tout en restant dans le domaine de la géométrie, à des situations qui échappent au lemme ordinaire.

\section{RÉFÉRENCES}

C. Berge, Espaces topologiques et fonctions multivoques, Dunod, Paris, 1959.

Ch. Bidard, Sraffa's Theory as a Combinatorics, mimeo, MODEM, Université de Paris-X-Nanterre, 1995.

Ch. Bidard et G. ERreygers, The Number and Type of Long Term Equilibria, mimeo, Universités de Paris-X-Nanterre et d'Anvers, 1995.

K. C. Border, Fixed Point Theorems with Applications to Economics and Game Theory, Cambridge University Press, Cambridge, 1985.

L. E. J. BRouwer, Über Abbildungen von Mannigfaltigkeiten, Mathematische Annalen, 1912, 71, p. 97-115.

E. Dierker, Two Remarks on the Number of Equilibria of an Economy, Econometrica, 1972, 40, p. 951-953.

F. J. Gould et J. W. Tolle, A Unified Approach to Complementarity in Optimisation, Discrete Mathematics, 1974,7 , p. 225-272.

B. Knaster, K. Kuratowski et S. Mazurkiewicz, Ein Beweis des Fixpunktsatze für n-dimensionale Simplexe, Fundamenta Mathematica, 1929, 14, p. 132-137.

C. E. Lemke, Bimatrix Equilibrium Points and Mathematical Programming, Management Science, 1965, 11, p. 681-689.

C. LE $\mathrm{V}_{\mathrm{AN}}$, Topological Degree and the Sperner Lemma, Journal of Optimization Theory and Applications, 1982, 37, p. 371-377.

M. LIPPI, I prezzi di produzione. Una saggio sulla teoria di Sraffa, II Mulino, Bologne, 1979.

H. E. Scarf, The Approximation of Fixed Points of a Continuous Mapping, SIAM Journal of Applied Mathematics, 1967, 15, p. 1328-1343. 
E. Sperner, Neur Beweis für die Invarianz der Dimensionszahl und des Gebietes, Abhandlungen an den mathematischen Seminar der Universität Hamburg, 1928, 6, p. $265-272$.

M. J. ToDD, A Generalized Complementary Pivoting Algorithm, Mathematical Programming, 1974, 6, p. 243-263.

H. TuY, Pivotal Methods for Computing Equilibrium Points: Unified Approach and New Restart Algorithm, Mathematical Programming, 1979, 16, p. 210-277.

M. Yoseloff, Topological Proofs of Some Combinatorial Theorems, Journal of Combinatorial Theory, 1974, 17, p. 95-111. 